\title{
Meromorphic solutions to certain class of differential equations in an angular domain
}

Fengrong Zhang ${ }^{1 *}$, Hui X ${ }^{1}$, Mengmeng Zhang ${ }^{1}$ and Daiwei Liang ${ }^{1}$

"Correspondence:

zhangfengrongsong@126.com

${ }^{1}$ College of Science, China

University of Petroleum (East China),

Qingdao, P.R. China

\section{Springer}

\begin{abstract}
In this paper, we study the admissible meromorphic solutions to the algebraic differential equation $f^{n} f^{\prime}+P_{n-1}(f)=u \mathrm{e}^{v}$ in an angular domain instead of the whole complex plane, where $P_{n-1}(f)$ is a differential polynomial in $f$ of degree $\leq n-1$ with small function coefficients, $u$ is a non-vanishing small function of $f$ and $v$ is an entire function. Herein, mainly, we are able to show that the equation does not admit any meromorphic solution $f$ under some conditions unless $P_{n-1}(f) \equiv 0$. Using this result, we are able to extend or generalize a well-known result of Hayman.
\end{abstract}

MSC: 30D35; 30D20; 30D30

Keywords: Nevanlinna theory on an angular domain; Admissible solution; Entire function; Differential equation

\section{Introduction}

In a recent paper, Liao and Ye considered meromorphic solutions $f$ to

$$
f^{n} f^{\prime}+Q_{d}(z, f)=u \mathrm{e}^{v}
$$

where $Q_{d}(z, f)$ denotes a differential polynomial in $f$ of degree $d$ with rational function coefficients. More precisely, they obtained the following result.

Theorem 1.1 ([1]) Let $Q_{d}(z, f)$ be a differential polynomial in $f$ of degree d with rational function coefficients. Suppose that $u$ is a non-zero rational function and $v$ is a non-constant polynomial. If $n \geq d+1$ and the differential equation (1.1) admits a meromorphic solution $f$ with finitely many poles, then $f$ has the following form:

$$
f=s e^{\nu /(n+1)} \quad \text { and } \quad Q_{d}(z, f) \equiv 0,
$$

where $s$ is a rational function with $s^{n}\left((n+1) s^{\prime}+v^{\prime} s\right)=(n+1) u$.

It becomes natural for us to ask the following question: Does the conclusion of Theorem 1.1 remains valid if the condition " $u$ is a non-zero rational function and $v$ is a nonconstant polynomial" is replaced by a weaker one, such as " $u$ and $v$ are entire functions" in the above theorem?

(c) The Author(s) 2019. This article is distributed under the terms of the Creative Commons Attribution 4.0 International License (http://creativecommons.org/licenses/by/4.0/), which permits unrestricted use, distribution, and reproduction in any medium, provided you give appropriate credit to the original author(s) and the source, provide a link to the Creative Commons license, and indicate if changes were made. 
In this paper, we consider a slightly more general case of Eq. (1.1), where $u$ and the coefficients of $Q_{d}(z, f)$ are meromorphic functions, not necessarily rational functions. Furthermore, $v$ is an entire function. In this case, we will show that there exists an angular domain $\Omega(\alpha, \beta)=\{z: \alpha \leq \arg z \leq \beta\}$ such that the conclusion of Theorem 1.1 remains valid, where $\alpha, \beta \in[0,2 \pi]$ and $0<\beta-\alpha \leq 2 \pi$.

The technique developed here will be different from what has been employed in [1]. Now, we first introduce the Nevanlinna theory on an angular domain, which can be found in Goldberg-Ostrovskii [2] and the references therein.

Herein let $f$ denote a non-constant meromorphic function on the closed angular domain $\bar{\Omega}(\alpha, \beta)=\Omega(\alpha, \beta) \cup\{0\} \cup\{\infty\}$, and let $k=\pi(\beta-\alpha)^{-1}, 1 \leq r<\infty$. Following GoldbergOstrovskii [2, pp. 23-26], we introduce the following notations [3]:

$$
\begin{aligned}
& A_{\alpha \beta}(r, f)=\frac{k}{\pi} \int_{1}^{r}\left(\frac{1}{t^{k}}-\frac{t^{k}}{r^{2 k}}\right)\left[\log ^{+}\left|f\left(t e^{i \alpha}\right)\right|+\log ^{+}\left|f\left(t e^{i \beta}\right)\right|\right] \frac{d t}{t} \\
& B_{\alpha \beta}(r, f)=\frac{2 k}{\pi r^{k}} \int_{\alpha}^{\beta} \log ^{+}\left|f\left(r e^{i \phi}\right)\right| \sin k(\phi-\alpha) d \phi ; \\
& C_{\alpha \beta}(r, f)=2 k \int_{1}^{r} c_{\alpha \beta}(t, f)\left(\frac{1}{t^{k}}+\frac{t^{k}}{r^{2 k}}\right) \frac{d t}{t}
\end{aligned}
$$

where

$$
c_{\alpha \beta}(r, f)=\sum_{1<\rho_{n} \leq r, \alpha \leq \varphi_{n} \leq \beta} \sin k\left(\varphi_{n}-\alpha\right)
$$

and the $\rho_{n} e^{i \varphi_{n}}$ are poles of $f$ counted according to their multiplicity. The function $C_{\alpha \beta}(r, f)$ is called the angular counting function of the poles of $f$ on $\bar{\Omega}(\alpha, \beta)$ and the Nevanlinna angular characteristic function is defined as $S_{\alpha \beta}(r, f)$ :

$$
S_{\alpha \beta}(r, f)=A_{\alpha \beta}(r, f)+B_{\alpha \beta}(r, f)+C_{\alpha \beta}(r, f) .
$$

Similarly, for any finite value $a$, we define here $A_{\alpha \beta}\left(r, f_{a}\right), B_{\alpha \beta}\left(r, f_{a}\right), C_{\alpha \beta}\left(r, f_{a}\right)$ and $S_{\alpha \beta}\left(r, f_{a}\right)$, where $f_{a}=1 /(f-a)$. In what follows, when there is no danger of confusion, next we omit the subscript of all the above notations and, respectively, use the notations $A\left(r, f_{a}\right), B\left(r, f_{a}\right)$, $C\left(r, f_{a}\right)$ and $S\left(r, f_{a}\right)$ instead of $A_{\alpha \beta}\left(r, f_{a}\right), B_{\alpha \beta}\left(r, f_{a}\right), C_{\alpha \beta}\left(r, f_{a}\right)$ and $S_{\alpha \beta}\left(r, f_{a}\right)$ for any finite value $a$.

In this paper, unless otherwise stated, we address a meromorphic function that is defined and meromorphic in the whole complex plane. It is assumed the reader is familiar with the basic theory of the Nevanlinna value distribution and its standard symbols and notations (see e.g., $[4,5])$.

For the order $\rho(f)=\infty$, we use the following concept of a proximate order as introduced in [6].

Theorem 1.2 ([6]) Let $B(r)$ be a positive and continuous function in $[0,+\infty)$, which satisfies $\limsup _{r \rightarrow \infty} \frac{\log B(r)}{\log r}=\infty$, then there exists a continuously differentiable function $\rho(r)$, which satisfies the following conditions.

(i) $\rho(r)$ is continuous and nondecreasing for $r>r_{0}\left(r_{0}>0\right)$ and tends to $+\infty$ as $r \rightarrow+\infty$. 
(ii) The function $U(r)=r^{\rho(r)}\left(r \geq r_{0}\right)$ satisfies the condition

$$
\lim _{r \rightarrow+\infty} \frac{\log U(R)}{\log U(r)}=1, \quad R=r+\frac{r}{\log U(r)}
$$

(iii)

$$
\limsup _{r \rightarrow+\infty} \frac{\log B(r)}{\log U(r)}=1
$$

We define $\rho(r)$ and $U(r)$ in Theorem 1.2 by the proximate order and type function of $B(r)$, respectively. For a transcendental meromorphic function $f$ of infinite order, we define its proximate order and type function as the proximate order and type function of $T(r, f)$. We denote $M(\rho(r))$ by the set of all meromorphic functions $f$ in the complex plane such that

$$
\limsup _{r \rightarrow \infty} \frac{\log T(r, f)}{\log U(r)}=1 .
$$

For the sake of simplicity, we use the Landau symbols $O(\cdots)$ and $o(\cdots)$ as $r \rightarrow \infty$, where and in what follows, $Q(r, f)$ is such a quantity that if the order $\rho(f)<\infty$, then $Q(r, f)=O(1)$, as $r \rightarrow \infty$; if the order $\rho(f)=\infty$, then $Q(r, f)=O(\log U(r))$. It is not necessarily the same for every occurrence in the context.

Throughout the paper, given a meromorphic function $f$, we define

$$
S_{f}:=\{h \mid \text { meromorphic, } S(r, h)=Q(r, f)\},
$$

as $r \rightarrow \infty$, possibly outside a set of $r$ values of finite linear measure.

In addition, we need the following concept (see, e.g., [7] and [8]).

Definition 1.3 Let $R(z, \omega)$ be rational in $\omega$ with meromorphic coefficients. A meromorphic solution $\omega$ of $\left(\omega^{\prime}\right)^{n}=R(z, \omega)$ is called admissible, if $S(r, a)=Q(r, \omega)$ holds for all coefficients $a$ of $R(z, \omega)$.

Of course, admissibility makes sense relative to any family of meromorphic functions, without any reference to differential equations.

Before proceeding further, we recall the following result. Recently, Liu-Lü-Yang considered the possible admissible solution to the following equation.

Theorem 1.4 ([9]) Let $p, q$ and $u$ be non-vanishing meromorphic functions in complex plane, $v$ be an entire function. Then the differential equation

$$
p f^{\prime}-q=u e^{v}
$$

has no transcendental meromorphic solutions in the complex plane.

Motivated by the preceding, as a continuation and further studies on some of the related results in the complex plane, we will state our main results in Sect. 3, which extend some results earlier; see, e.g., [10-13] and the references therein. 


\section{Preliminary lemmas}

To prove our results, the following lemmas are needed.

Lemma 2.1 ([2, pp. 23-26] and [2, Theorem 3.1]) Letf be a non-constant meromorphic function and $\Omega(\alpha, \beta)$ be a domain, where $0<\beta-\alpha \leq 2 \pi$. Then, for an arbitrary complex number $a \in \mathbb{C}$ and any integer $k \geq 0$, we have

$$
\begin{aligned}
& S\left(r, \frac{1}{f-a}\right)=S(r, f)+O(1), \\
& S\left(r, f^{(k)}\right) \leq 2^{k} S(r, f)+Q(r, f),
\end{aligned}
$$

and

$$
A\left(r, \frac{f^{(k)}}{f}\right)+B\left(r, \frac{f^{(k)}}{f}\right)=Q(r, f)
$$

Lemma 2.2 ([2, Theorem 3.3]) Let $f$ be a meromorphic function. Then, for arbitrary $q$ distinct values $a_{j} \in \mathbb{C} \cup\{\infty\}(1 \leq j \leq q)$,

$$
(q-2) S(r, f) \leq \sum_{j=1}^{q} \bar{C}\left(r, \frac{1}{f-a_{j}}\right)+Q(r, f),
$$

where $\bar{C}\left(r, \frac{1}{f-a_{j}}\right)$ is the reduced form of $C\left(r, \frac{1}{f-a_{j}}\right)$.

Lemma 2.3 ([2]) Let $f$ be a meromorphic function. By $D(r, f)$, we denote any of the characteristics $A(r, f), B(r, f)$, and $C(r, f)$. Then

$$
\begin{aligned}
& D(r, f+g) \leq D(r, f)+D(r, g)+\log 2, \\
& D(r, f \cdot g) \leq D(r, f)+D(r, g),
\end{aligned}
$$

and

$$
D\left(r, f^{k}\right)=k D(r, f)
$$

for any positive integer $k$.

Lemma 2.4 Letf be a meromorphic solution of

$$
f^{n} P_{1}(z, f)=P_{2}(z, f)
$$

where $P_{1}(z, f)$ and $P_{2}(z, f)$ are polynomials in $f$ and its derivatives with meromorphic coefficients $\left\{a_{\lambda} \mid \lambda \in I\right\}$ such that $S\left(r, a_{\lambda}\right)=Q(r, f)$ for all $r \in I$. If the total degree of $Q(z, f)$ as a polynomial in $f$ and its derivatives is less than or equal to $n$, then

$$
A(r, P(r, f))+B(r, P(r, f))=Q(r, f) .
$$

We omit the proof of Lemma 2.4, since it is similar to the proof of Clunie's theorem [4, p. 68, Lemma 3.3] and $[14,15]$ in the plane. 


\section{Main results}

In this section, we give our main results as follows.

Theorem 3.1 Let $f$ be a transcendental meromorphic function, $P_{n-1}(f)$ be a differential polynomial in $f$ such that its coefficients are in $S_{f}$ and $\operatorname{deg} P_{n-1}(f) \leq n-1$. Assume further that $v$ is a non-constant entire function of finite order and $u\left(u \in S_{f}\right)$ is a meromorphic function. Then, for any positive integer $n$, and any $\varepsilon(0<\varepsilon<\pi / 2)$, there exists a direction $\arg z=\theta$ such that the equation

$$
f^{n} f^{\prime}+P_{n-1}(f)=u \mathrm{e}^{v}
$$

does not admit any non-constant meromorphic solution $f$ on the domain $\Omega(\theta-\varepsilon, \theta+\varepsilon)$ with $C(r, f)=Q(r, f)$ unless $P_{n-1}(f)=0$. Moreover, if (3.1) admits a meromorphic solution $f$ on $\Omega(\theta-\varepsilon, \theta+\varepsilon)$ with $C(r, f)=Q(r, f)$, then (3.1) will become $f^{n} f^{\prime}=u \mathrm{e}^{v}$ and $f$ must have the form $f=\psi \exp (v /(n+1))$ as the only possible admissible solution of $(3.1)$, where $\psi$ is in $S_{f}$.

Proof First of all, we suppose that $f \in M(\rho(r))$. In this case, we show that $f^{n} f^{\prime}+P_{n-1}(f)$ cannot be $S_{f}$. Otherwise, from $C(r, f)=Q(r, f)$ and Lemma 2.4, we get $A\left(r, f^{\prime}\right)+B\left(r, f^{\prime}\right)=$ $Q(r, f)$ and then $S\left(r, f^{\prime}\right)=Q(r, f)$. A contradiction $S(r, f)=Q(r, f)$ now follows by relying on Theorem 2.6.5 in [16, p. 91]. Thus, for any meromorphic function $f$ under the condition: $C(r, f)=Q(r, f)$,

$$
S\left(r, f^{n} f^{\prime}+P_{n-1}(f)\right) \neq Q(r, f) .
$$

Therefore (3.2) shows that $u \mathrm{e}^{v}$ is not in $S_{f}$.

It is well known that a meromorphic function $f \in M(\rho(r))$ has at least one Borel direction $\arg z=\theta$ of order $\rho(r)$. In the following, we prove that the direction $\arg z=\theta$ satisfies the theorem. For any $\varepsilon(0<\varepsilon<\pi / 2)$, then

$$
\limsup _{r \rightarrow \infty} \frac{\log S(r, f)}{\log r^{\rho(r)}}=1
$$

holds on $\Omega(\theta-\varepsilon, \theta+\varepsilon) \cup\{0\} \cup\{\infty\}$.

To prove the theorem, we first assume that $P_{n-1}(f) \not \equiv 0$. By examining carefully the proof of the Milloux estimate in [4, p. 55], we deduce from the assumption $C(r, f)=Q(r, f)$ and (3.1) that

$$
S\left(r, \mathrm{e}^{v}\right) \leq(n+1) S(r, f)+Q(r, f)
$$

which shows that $v$ and $v^{\prime}$ are in $S_{f}$.

Taking the logarithmic derivative on both sides of (3.1) yields

$$
\frac{n f^{n-1}\left(f^{\prime}\right)^{2}+f^{n} f^{\prime \prime}+P_{n-1}^{\prime}(f)}{f^{n} f^{\prime}+P_{n-1}(f)}=\frac{u^{\prime}}{u}+v^{\prime}
$$

which gives the equality

$$
-\left(\frac{u^{\prime}}{u}+v^{\prime}\right) f^{n} f^{\prime}+n f^{n-1}\left(f^{\prime}\right)^{2}+f^{n} f^{\prime \prime}=\left(\frac{u^{\prime}}{u}+v^{\prime}\right) P_{n-1}(f)-P_{n-1}^{\prime}(f) .
$$


Now, we set

$$
\varphi=-\left(\frac{u^{\prime}}{u}+v^{\prime}\right) f f^{\prime}+n\left(f^{\prime}\right)^{2}+f^{\prime \prime}
$$

It follows by (3.3) that

$$
f^{n-1} \varphi=\left(\frac{u^{\prime}}{u}+v^{\prime}\right) P_{n-1}(f)-P_{n-1}^{\prime}(f):=P_{n-1}^{*}(f) .
$$

Clearly, $P_{n-1}^{*}(f)$ is a differential polynomial in $f$ with $\operatorname{deg} P_{n-1}^{*}(f) \leq n-1$. First of all, let us show that $\varphi \neq \equiv$. Assume the contrary, that is, $\varphi \equiv 0$. Then $P_{n-1}^{*}(f) \equiv 0$, and accordingly (3.5) gives $B P_{n-1}(f) \equiv u \mathrm{e}^{v}$ with a constant $B$. Since $f$ is a non-constant meromorphic function, (3.1) shows that $B \neq 1$, and

$$
f^{n} f^{\prime}=(B-1) P_{n-1}(f)
$$

which together with Lemma 2.4 implies $A\left(r, f^{\prime}\right)+B\left(r, f^{\prime}\right)=Q(r, f)$. Thereby we have $S\left(r, f^{\prime}\right)=Q(r, f)$ from the assumption $C(r, f)=Q(r, f)$. This is a contradiction. Hence $\varphi \not \equiv 0$. Moreover, applying Lemma 2.4 to (3.5) again, we can derive $A(r, \varphi)+B(r, \varphi)=Q(r, f)$ and so $S(r, \varphi)=Q(r, f)$. That is $\varphi \in S_{f}$.

Note that (3.4) can be represented in the form

$$
\frac{\varphi}{f^{2}}=-\left(\frac{u^{\prime}}{u}+v^{\prime}\right) \frac{f^{\prime}}{f}+n\left(\frac{f^{\prime}}{f}\right)^{2}+\frac{f^{\prime \prime}}{f}
$$

which and Lemma 2.1 yield $A\left(r, \frac{\varphi}{f^{2}}\right)+B\left(r, \frac{\varphi}{f^{2}}\right)=Q(r, f)$. Thus, we obtain

$$
A\left(r, \frac{1}{f}\right)+B\left(r, \frac{1}{f}\right)=Q(r, f)
$$

On the other hand, it is clear that (3.4) shows that

$$
C_{(2}\left(r, \frac{1}{f}\right) \leq C\left(r, \frac{1}{\varphi}\right)+Q(r, f) \leq S(r, \varphi)+Q(r, f)=Q(r, f)
$$

which implies that the zeros of $f$ are mainly simple zeros on $\Omega(\theta-\varepsilon, \theta+\varepsilon)$. This observation will be repeatedly used later on. Thus, by (3.6) and the definition of $S(r, f)$, we obtain

$$
S(r, f)=C\left(r, \frac{1}{f}\right)+Q(r, f)=C_{1)}\left(r, \frac{1}{f}\right)+Q(r, f),
$$

where in $C_{1)}(r, 1 / f)$ only the simple zeros of $f$ are to be considered on $\Omega(\theta-\varepsilon, \theta+\varepsilon)$.

Without loss of generality we may assume that $z_{0}$ be a simple zero of $f$ such that $u\left(z_{0}\right) \neq 0$. Then we find by (3.4) that

$$
n\left(f^{\prime}\right)^{2}\left(z_{0}\right)=\varphi\left(z_{0}\right)
$$


If $\varphi$ is a constant, and set

$$
h(z)=\frac{f^{\prime}(z)-\sqrt{\frac{\varphi}{n}}}{f(z)} .
$$

Obviously $h \not \equiv 0$ since $f$ is transcendental. It follows by (3.6), (3.8), (3.9) and Lemma 2.1 that

$$
A(r, h)+B(r, h)=Q(r, f) .
$$

From (3.7) and (3.9), we arrive at $C(r, h)=Q(r, f)$, which and (3.10) show that $h \in S_{f}$. Now, we rewrite (3.9) as

$$
f^{\prime}(z)=h(z) f(z)+\sqrt{\frac{\varphi}{n}} .
$$

By (3.11) and (3.4), we obtain

$$
\left[(n+1) h^{2}+h^{\prime}-h\left(\frac{u^{\prime}}{u}+v^{\prime}\right)\right] f^{2}+\left[2 n h-\left(\frac{u^{\prime}}{u}+v^{\prime}\right)\right] \sqrt{\frac{\varphi}{n}} f+h \sqrt{\frac{\varphi}{n}}=0,
$$

this and Lemma 2.3 (or [2, p. 33, Theorem 6.3]) imply $h \sqrt{\frac{\varphi}{n}} \equiv 0$, a contradiction. Thus $\varphi^{\prime} \not \equiv 0$. Again, by taking the logarithmic derivative on both sides of (3.4), we conclude

$$
\varphi^{\prime}=-t^{\prime} f^{\prime}-t\left(f^{\prime}\right)^{2}-t f^{\prime \prime}+(2 n+1) f^{\prime} f^{\prime \prime}+f^{\prime \prime \prime},
$$

where $t=\frac{u^{\prime}}{u}+v^{\prime}$. By (3.12) and (3.8), we see that a simple zero $z_{0}$ of $f(z)$ such that $u\left(z_{0}\right) \neq 0$, is a zero of $(2 n+1) \varphi f^{\prime \prime}(z)-\left(t \varphi+n \varphi^{\prime}\right) f^{\prime}(z)$.

Two cases will now be considered below, depending on whether or not $(2 n+1) \varphi f^{\prime \prime}(z)-$ $\left(t \varphi+n \varphi^{\prime}\right) f^{\prime}(z)$ vanishes identically.

Case 1. $(2 n+1) \varphi f^{\prime \prime}(z)-\left(t \varphi+n \varphi^{\prime}\right) f^{\prime}(z) \not \equiv 0$. Set

$$
g(z)=\frac{(2 n+1) \varphi f^{\prime \prime}(z)-\left(t \varphi+n \varphi^{\prime}\right) f^{\prime}(z)}{f(z)} .
$$

Trivially, $g(z) \in S_{f}$. Thus, we have

$$
f^{\prime \prime}=\frac{g}{(2 n+1) \varphi} f+\frac{t \varphi+n \varphi^{\prime}}{(2 n+1) \varphi} f^{\prime}:=s_{1} f+s_{2} f^{\prime}
$$

and

$$
f^{\prime \prime \prime}=\left(s_{1}^{\prime}+s_{1} s_{2}\right) f+\left(s_{1}+s_{2}^{\prime}+s_{2}^{2}\right) f^{\prime} .
$$

It follows by (3.14), (3.13), (3.12) and (3.4) that

$$
\begin{aligned}
& \left((2 n+1) s_{1}-t^{\prime}-t s_{2}+s_{1}+s_{2}^{\prime}+s_{2}^{2}+t \frac{\varphi^{\prime}}{\varphi}-s_{2} \frac{\varphi^{\prime}}{\varphi}\right) f^{\prime} \\
& +\left(s_{1}^{\prime}+s_{1} s_{2}-t s_{1}-s_{1} \frac{\varphi^{\prime}}{\varphi}\right) f=0 .
\end{aligned}
$$


In this case, (3.15) and (3.7) imply

$$
s_{1}^{\prime}+s_{1} s_{2}-t s_{1}-s_{1} \frac{\varphi^{\prime}}{\varphi} \equiv 0 .
$$

Since $g \not \equiv 0$, we know that $s_{1} \not \equiv 0$. Thus, we can rewrite the above equation as

$$
\frac{s_{1}^{\prime}}{s_{1}}+s_{2}-t-\frac{\varphi^{\prime}}{\varphi} \equiv 0
$$

hence we have $(2 n+1) \log s_{1}=2 n(\log u+v)+(3 n+1) \log \varphi+L$ with a constant $L$, which implies $\left(u \mathrm{e}^{v}\right)^{2 n} \mathrm{e}^{L} \varphi^{3 n+1}=s_{1}^{2 n+1}$, we see that $u \mathrm{e}^{v}$ is in $S_{f}$, this contradicts (3.2).

Case 2. $(2 n+1) \varphi f^{\prime \prime}(z)-\left(t \varphi+n \varphi^{\prime}\right) f^{\prime}(z) \equiv 0$. In this case, we conclude

$$
f^{\prime \prime}=\xi f^{\prime}
$$

with $\xi=\frac{n \varphi^{\prime}}{(2 n+1) \varphi}+\frac{t}{2 n+1}$. Thus (3.16) gives

$$
f^{\prime \prime \prime}=\left(\xi^{\prime}+\xi^{2}\right) f^{\prime}
$$

It follows by (3.17), (3.16), (3.12) and (3.4) that

$$
\left(\xi^{\prime}+\xi^{2}\right) f^{\prime}=\left(t^{\prime}-t \frac{\varphi^{\prime}}{\varphi}\right) f^{\prime}+\left(t+\frac{\varphi^{\prime}}{\varphi}\right) \xi f^{\prime} .
$$

Thus, we have

$$
\xi^{\prime}-t^{\prime} \equiv-\xi(\xi-t)+(\xi-t) \frac{\varphi^{\prime}}{\varphi} .
$$

If $\xi-t \equiv 0$, then, by the definitions of $t$ and $\xi$, we see that $\left(u \mathrm{e}^{\nu}\right)^{2}=\eta \varphi, \eta$ is a constant. So $u \mathrm{e}^{v}$ is in $S_{f}$, this contradicts (3.2). Hence $\xi-t \not \equiv 0$. In this case, again, by (3.18), we obtain $(2 n+1) \log (\xi-t)=n \log \varphi+\log u+v+\tau$ with a constant $\tau$. This gives $u \mathrm{e}^{v} \in S_{f}$, this also contradicts (3.2).

This completes the proof of our conclusion, namely $f^{n} f^{\prime}+P_{n-1}(f)=u \mathrm{e}^{v}$ does not admit any meromorphic solution $f$ on $\Omega(\theta-\varepsilon, \theta+\varepsilon)$ with $C(r, f)=Q(r, f)$ unless $P_{n-1}(f) \equiv 0$.

Assume that $\rho(f)<+\infty$. Note $v$ is a non-constant entire function, so we have $\rho(f)>0$. Let $\sigma$ satisfy $0<\sigma<\rho(f)$. For a given angular domain $\Omega(\alpha, \beta), \beta-\alpha=\frac{\pi}{\sigma}$. Without loss of generality, we may assume that $f$ has at least one Borel direction in the angular domain $\Omega(\alpha+\varepsilon, \beta-\varepsilon)(0<\varepsilon<\pi / 2)$. Hence, there exists a finite complex number $a$ such that

$$
\limsup _{r \rightarrow \infty} \frac{\log n(r, \Omega(\alpha+\varepsilon, \beta-\varepsilon), f=a)}{\log r}>\sigma=\frac{\pi}{\beta-\alpha} .
$$

The Hadamard factorization theorem and (3.1) give $f=\psi \exp (v /(n+1))$, where $\psi$ is in $S_{f}$. Then the conclusion of the theorem follows. The proof is finished.

Corollary 3.2 Let $f$ be a transcendental meromorphic function, $P_{n-1}(f)\left(P_{n-1}(0) \neq 0\right)$ denote a differential polynomial in $f$ with its coefficients are in $S_{f}$ and $\operatorname{deg} P_{n-1}(f) \leq n-1$. Then, for any positive integer $n$, and any $\varepsilon(0<\varepsilon<\pi / 2)$, there exists a direction $\arg z=\theta$ such that $f^{n} f^{\prime}+P_{n-1}(f)$ has infinitely many zeros on $\Omega(\theta-\varepsilon, \theta+\varepsilon)$ with $C(r, f)=Q(r, f)$. 
Remark 3.3 Take $f(z)=\mathrm{e}^{z}+\mathrm{e}^{-z}$, and $P_{0}(f)=-2$. Obviously, $f f^{\prime \prime}+P_{0}(f)=\mathrm{e}^{2 z}+\mathrm{e}^{-2 z}$ has infinitely many zeros.

Based on Corollary 3.2 and Remark 3.3, we present the following more general conjecture; see, e.g., [3] and [17].

Conjecture 3.4 Letf be a transcendental meromorphic function, and assume further that $P_{n-1}(f)$ is a differential polynomial inf with its coefficients are in $S_{f}$ and $\operatorname{deg} P_{n-1}(f) \leq n-1$ such that $P_{n-1}(0) \neq 0$. Then, for any positive integers $n, k$, and any $\varepsilon(0<\varepsilon<\pi / 2)$, there exists a direction $\arg z=\theta$ such that

$$
C\left(r, \frac{1}{f^{n} f^{(k)}+P_{n-1}(f)}\right) \neq Q(r, f)
$$

on $\Omega(\theta-\varepsilon, \theta+\varepsilon)$ with $C(r, f)=Q(r, f)$.

Remark 3.5 We will give some examples below to show that the restricted conditions in Corollary 3.2 are sharp.

Example 3.6 Take $f(z)=\mathrm{e}^{\mathrm{e}^{z}}-1$, and $P_{2}(f)=2 \mathrm{e}^{z} f^{2}+3 \mathrm{e}^{z} f+\mathrm{e}^{z}$. Obviously, $\mathrm{e}^{z} \in S_{f}$, and $f^{2} f^{\prime}+$ $P_{2}(f)=\mathrm{e}^{z} \mathrm{e}^{3 \mathrm{e}^{z}}$ has no zeros on $\Omega(\theta-\varepsilon, \theta+\varepsilon)$.

Example 3.7 Let $f(z)=\mathrm{e}^{z^{2}}-1$, and $P_{1}(f)=2 z f+2 z$. Obviously, $f^{\prime}+P_{1}(f)=2 z \mathrm{e}^{2 z^{2}}$ has only one zero on $\Omega(\theta-\varepsilon, \theta+\varepsilon)$.

Examples 3.6 and 3.7 tell us that the conclusion of Corollary 3.2 is false, if we replace the restricted condition " $\operatorname{deg} P_{n-1}(f) \leq n-1$ " by “ $\operatorname{deg} P_{n}(f) \leq n$ ".

Example 3.8 If we take $f(z)=z^{2} \mathrm{e}^{z}, P_{2}(f)=f^{\prime}-\left(\frac{2}{z}+1\right) f^{2}$, then $2 / z+1 \in S_{f}$, and $f^{3} f^{\prime}+P_{2}(f)=$ $(2+z) z^{7} \mathrm{e}^{4 z}$ has only finitely many zeros on $\Omega(\theta-\varepsilon, \theta+\varepsilon)$.

Example 3.8 shows that the restricted condition " $P_{n-1}(0) \neq 0$ " in Corollary 3.2 is necessary.

Corollary 3.2 may be false if the condition " $C(r, f)=Q(r, f)$ " is violated. There is no difficulty in showing that Example 3.9 below is a counterexample.

Example 3.9 We take $\alpha=-\pi, \beta=\pi, f(z)=\frac{\mathrm{e}^{z}}{\mathrm{e}^{z}-1}, P_{1}(f)=\frac{3}{2} f^{\prime \prime}+\frac{3}{2} f^{\prime}+f-1$.

Indeed, a calculation yields $C(r, 1 / f)=0, C(r, 1 /(f-1))=0$. It follows by Lemma 2.2 that $C(r, f)=S(r, f)+Q(r, f)$. Further, we would like to mention that $f^{2} f^{\prime}+P_{1}(f)=-\frac{1}{\left(\mathrm{e}^{z}-1\right)^{4}}$ has no zeros on $\Omega(\theta-\varepsilon, \theta+\varepsilon)$.

By noting that $f$ in the above counterexample (Example 3.9) satisfies " $\bar{C}(r, f)=S(r, f)+$ $Q(r, f)$, we can see that it would be interesting if one could characterize all the solutions on some $\Omega(\theta-\varepsilon, \theta+\varepsilon)$ to the equation

$$
f^{n} f^{(k)}+P_{n-1}(f)=u \mathrm{e}^{v}
$$

with $\bar{C}(r, f) \neq S(r, f)+Q(r, f)$, where $n, k$ are positive integers. 
From Theorem 3.1, we also obtain the following result, which complements the corresponding results in [1].

Theorem 3.10 Let $f \in M(\rho(r))$ of finite order with $C(r, f)=Q(r, f), q_{m}(f)=b_{m} f^{m}+\cdots+$ $b_{1} f+b_{0}$ a polynomial of degree $m$ with $b_{j} \in S_{f}(j=0,1, \ldots, m)$, and let $n$ be an integer with $n \geq m+1$. Then, for any $\varepsilon(0<\varepsilon<\pi / 2)$, there exists a direction $\arg z=\theta$ such that $f^{\prime} f^{n}+q_{m}(f)$ assumes every function $\gamma \in S_{f}$ infinitely many times on $\Omega(\theta-\varepsilon, \theta+\varepsilon)$, except for a possible function $b_{0}=q_{m}(0)$. On the other hand, if $f^{\prime} f^{n}+q_{m}(f)$ assumes $b_{0}=q_{m}(0)$ finitely many times on $\Omega(\theta-\varepsilon, \theta+\varepsilon)$, then $q_{m}(z) \equiv b_{0}$.

Proof This theorem can be proved in the same manner as that in the proof of Theorem 3.1, so it is omitted here.

\section{Conclusions}

Using the different and much simpler proofs, this paper provides two main results on $\Omega(\alpha, \beta)$, which extend the main results that were derived in [1]. To bring about our results from the more general hypotheses without complicated calculations will probably be the most interesting feature of this note. And then some examples show that the restrict conditions are necessary. Finally, one more general conjecture was posed in this note.

\section{Acknowledgements}

The authors would like to thank the referee for his/her several important suggestions and for pointing out some errors in our original manuscript. Professor Weiran Lü also gave some suggestions as regards the manuscript.

\section{Funding}

This work was supported by the Fundamental Research Funds for the Central Universities (No. 18CX02045A) and the National Natural Science Foundation of China (Nos. 11602305, 11601521).

\section{Competing interests}

The authors declare that they have no competing interests.

\section{Authors' contributions}

All authors contributed in drafting this manuscript. All authors read and approved the final manuscript.

\section{Publisher's Note}

Springer Nature remains neutral with regard to jurisdictional claims in published maps and institutional affiliations.

Received: 7 September 2018 Accepted: 27 January 2019 Published online: 05 March 2019

\section{References}

1. Liao, L.W., Ye, Z.: On solutions to nonhomogeneous algebraic differential equations and their application. J. Aust. Math. Soc. 97, 391-403 (2014)

2. Goldberg, A.A., Ostrovskii, I.V.: Value Distribution of Meromorphic Functions. Translations of Mathematical Monographs, vol. 236. Am. Math. Soc., Providence (2008) Translated from the 1970 Russian original by Mikhail Ostrovskii

3. Yang, L., Yang, C.C.: Angular distribution of values of ff'. Sci. China Ser. A 37, 284-294 (1994)

4. Hayman, W.K.: Meromorphic Functions. Clarendon, Oxford (1964)

5. Yang, C.C., Yi, H.X.: Uniqueness Theory of Meromorphic Functions. Science Press, Beijing (2003)

6. Chuang, C.T.: Differential Polynomials of Meromorphic Functions. Beijing Normal University Press, Beijing (1999) (in English)

7. Ishizaki, K., Yanagihara, N.: On admissible solutions of algebraic differential equations. Funkc. Ekvacioj 38, 433-442 (1995)

8. Laine, l.: Nevanlinna Theory and Complex Differential Equations. de Gruyter, Berlin (1993)

9. Liu, N.N., Lü, W.R., Yang, C.C.: On the meromorphic solutions of certain class of nonlinear differential equations. J. Inequal. Appl. 2015, 149 (2015)

10. Langley, J.K.: On the zeros of $f^{\prime \prime}-b$. Results Math. 44, 130-140 (2003)

11. Lü, W.R., Liu, N.N., Yang, C.C., Zhuo, C.P.: Notes on the value distribution of $\mathrm{ff}^{(k)}-$ b. Kodai Math. J. 39, 500-509 (2016)

12. Mues, E.: Über ein Problem von Hayman. Math. Z. 164, 239-259 (1979)

13. Zhang, J., Liao, L.: Admissible meromorphic solutions of algebraic differential equations. J. Math. Anal. Appl. 397 225-232 (2013) 
14. Clunie, J.: On integral and meromorphic functions. J. Lond. Math. Soc. 37, 17-27 (1962)

15. Yang, C.C., Ye, Z.: Estimates of the proximity function of differential polynomials. Proc. Jpn. Acad., Ser. A, Math. Sci. 83, 50-55 (2007)

16. Zheng, J.H.: Value Distribution of Meromorphic Functions. Tsinghua University Press, Beijing; Springer, Berlin (2010)

17. Yang, C.C., Yang, L., Wang, Y.F.: On the zeros of $\left(f^{(k)}\right)^{n} f-a$. Chin. Sci. Bull. 38, 2125-2128 (1993)

Submit your manuscript to a SpringerOpen ${ }^{\circ}$ journal and benefit from:

- Convenient online submission

- Rigorous peer review

- Open access: articles freely available online

- High visibility within the field

- Retaining the copyright to your article

Submit your next manuscript at $\gg$ springeropen.com 